\title{
Vocalization as an indicator of estrus climax in Holstein heifers during natural estrus and superovulation
}

\author{
Volker Röttgen, ${ }^{\dagger} †$ Frank Becker,† Armin Tuchscherer,† Christine Wrenzycki,§ Sandra Düpjan, ${ }^{*}$ \\ Peter C. Schön, ${ }^{*}$ and Birger Puppe*\# ${ }^{1}$ \\ *Institute of Behavioural Physiology, \\ †Institute of Reproductive Biology, \\ ‡Institute of Genetics and Biometry, Leibniz Institute for Farm Animal Biology (FBN), D-18196 Dummerstorf, Germany \\ $\S$ Chair of Molecular Reproductive Medicine, Clinic for Veterinary Obstetrics, Gynecology and Andrology, Faculty of Veterinary Medicine, \\ Justus-Liebig-University Giessen, D-35392 Giessen, Germany \\ \#Behavioural Sciences, Faculty of Agricultural and Environmental Sciences, University of Rostock, D-18059 Rostock, Germany
}

\section{ABSTRACT}

The reliable detection of estrus is an important scientific and practical challenge in dairy cattle farming. Female vocalization may indicate reproductive status, and preliminary evidence suggests that this information can be used to detect estrus in dairy cattle. The aim of this study was to associate the changes in the vocalization rate of dairy heifers with behavioral estrus indicators as well as test the influence of the type of estrus (natural estrus vs. superovulation-induced estrus). We analyzed 6 predefined estrus-related behavior patterns (standing to be mounted, head-side mounting, active mounting, chin resting, being mounted while not standing, and active sniffing in the anogenital region) and vocalization rates in the peri-estrus period (day of estrus $\pm 1 \mathrm{~d}$ ) of 12 German Holstein heifers using audio-visual recordings. Each heifer was observed under natural estrus and a consecutive superovulation induced by FSH and cloprostenol. Estrus was determined by behavioral patterns and confirmed by clinical examination (vaginoscopy and ultrasound imaging of the ovaries) as well as by the concentration of peripheral progesterone. Estrus behavior and vocalization rates were analyzed in 3-h intervals (an average of 19 intervals for each heifer), and an estrus score was calculated based on the 6 behaviors. The interval with the highest estrus score (I0) was considered the estrus climax. We demonstrated similar time courses for the estrus score and vocalization rate independent of estrus type. However, in natural estrus, the maximum vocalization rate $( \pm \mathrm{SE})$ occurred in the interval before estrus climax (I-1; $42.58 \pm 21.89)$ and was significantly higher than that in any other interval except estrus climax (I0; $27.58 \pm 9.76)$. During natural

Received June 29, 2017.

Accepted November 21, 2017.

${ }^{1}$ Corresponding author: puppe@fbn-dummerstorf.de estrus, the vocalization rate was significantly higher within the interval before estrus climax (I-1; $42.58 \pm$ 21.89 vs. $11.58 \pm 5.51)$ than under superovulation. The results underscore the potential use of vocalization rate as a suitable indicator of estrus climax in automated estrus detection devices. Further studies and technical development are required to record and process individual vocalization rates.

Key words: estrus detection, estrus behavior, bioacoustics, cattle

\section{INTRODUCTION}

Estrus detection is critical for reproductive performance and therefore of great concern in the dairy cattle industry. Effectively identifying cows in estrus is one of the everyday challenges faced by dairy farmers.

Visual detection of estrus remains one of the most reliable methods of identification, but it involves high staff costs (Rae et al., 1999; Rorie et al., 2002; Rutten et al., 2014) because the herd must be observed up to 3 times daily for 20 to $30 \mathrm{~min}$. VanVliet and VanEerdenburg (1996) introduced an estrus scoring system based on behavioral observations, in which different behaviors are weighed according to their specificity to indicate estrus (Table 1) and then aggregated into a single score. The need for a reliable but more costeffective method to identify the optimal insemination time for dairy cattle has promoted the development of automatic estrus detection devices. Typically, such devices only use one parameter to detect estrus, but some authors suggest that combinations of parameters could be more effective (Reith and Hoy, 2012). The following combinations of indicators have been proven to increase estrus detection rates: (1) visual estrus detection, activity measurements, and mounting detection (Peralta et al., 2005); (2) visual estrus detection with either a hormone-treated steer or tail paint and activity measurements combined with milk data (yield, 
Table 1. The estrus behaviors and their definition ${ }^{1}$

\begin{tabular}{|c|c|c|}
\hline Behavior & Definition & $\begin{array}{l}\text { Estrus score } \\
\text { points }\end{array}$ \\
\hline Standing & Standing to be mounted: accepting the mounting of another heifer. & 100 \\
\hline Mounting head & Mounting with the claws above the carpal joint level. & 45 \\
\hline Chin rest & $\begin{array}{l}\text { The chin of the observed heifer resting on the dorsal line of a herd member, between the withers and } \\
\text { the tail, and not more than } 10 \mathrm{~cm} \text { along the back line. }\end{array}$ & 15 \\
\hline
\end{tabular}

${ }^{1}$ The estrus score points are the factors that were multiplied by the recorded count of the behavior per interval (according to Van Vliet and VanEerdenburg, 1996).

temperature, and conductivity; Firk et al., 2002); and (3) activity, rumination time, and BW (Reith and Hoy, 2012). The efficacy and accuracy of automated estrus detection devices might also be increased by incorporating additional, yet unstudied parameters that are directly or indirectly related to estrus or estrus behavior. Vocalization rate is one such behavioral trait with promising characteristics for estrus detection (Schön et al., 2007; Dreschel et al., 2014), but no studies have described a time course for vocalization rates relative to established behavioral signs of estrus in dairy cows.

Changes in female vocalization that are dependent on the sexual cycle have been almost completely neglected in behavioral and bio-acoustic research. Some data are available from studies of humans (Bryant and Haselton, 2009), mammals (Charlton et al., 2010), birds (Langmore and Davies, 1997; Langmore et al., 1996), and amphibians (Tobias et al., 1998), and these studies describe different functions of female vocalizations that contribute to specific mating behaviors. In some species, vocalizations or specific elements of the vocal repertoire increase during estrus [e.g., African elephant (Loxodonta africana), Leong et al., 2003; Long-Evans rats (Rattus norvegicus), Matochik et al., 1992].

In cattle, vocalization alterations can occur in the context of pain (Stookey et al., 1996) or handling (Grandin, 2001), and some investigators have decoded the information conveyed by specific vocalizations (e.g., individual recognition, delayed milking, or hunger; Ikeda and Ishii, 2008; Jahns, 2008). In previous studies, we demonstrated that vocalization rates increase in heifers in estrus, both in tiestalls and group housing (Schön et al., 2007; Dreschel et al., 2014). However, none of these studies have demonstrated the temporal course of the vocalization rate in relation to estrus behavior, which is necessary for the development of automated estrus detection systems.

In cattle breeding, different hormonal treatments are commonly used to enhance fertility and breeding progress as well as reduce the number of nonpregnant days. Superovulation is a hormonal treatment that affects ovarian function by increasing the number of recruited primordial follicles and growth of dominant follicles, and it changes the number of ovulations and the concentrations of peripheral hormones (Roberge et al., 1995). Moreover, evidence indicates that superovulation not only increases the serum estradiol level but estrus behavior as well (Jezierski, 1993; Jimenez et al., 2011). Superovulation in cattle can be achieved by the administration of FSH in a descending dosage and induced luteolysis via $\mathrm{PGF}_{2 \alpha}$.

The primary aim of our study was to characterize the variation in vocalization during estrus in relation to the behaviors that are typically used for visual estrus detection, and we also analyzed the temporal association between ovulation and vocalization. The secondary aim of this study was to explore if the change in estrus behavior under superovulation treatment also affects the temporal vocalization pattern.

\section{MATERIALS AND METHODS}

In this study, we examined 12 German Holstein heifers in 3 groups of 4 animals each during natural estrus (NAT) and a subsequent superovulation treatment (SUP). The animals were housed in the experimental pen on the grounds of the Experimental Facility for Cattle at the Leibniz Institute of Farm Animal Biology, Dummerstorf, Germany. The experimental pen was a solitary building that was partially sound insulated, meaning that environmental noise was reduced and sound reflections within the pen were minimized by sound-dampening wall padding. Each group was continuously housed in the pen throughout the experimental period of approximately $60 \mathrm{~d}$ between March and November. The $5 \times 10 \mathrm{~m}$ pen was equipped with rubber flooring and wood shavings as bedding material, 3 drinking spots, and a self-locking feed barrier.

The average age of the heifers was 15.6 mo (range of 13.5-17.6 mo). Before being entered into the study, 


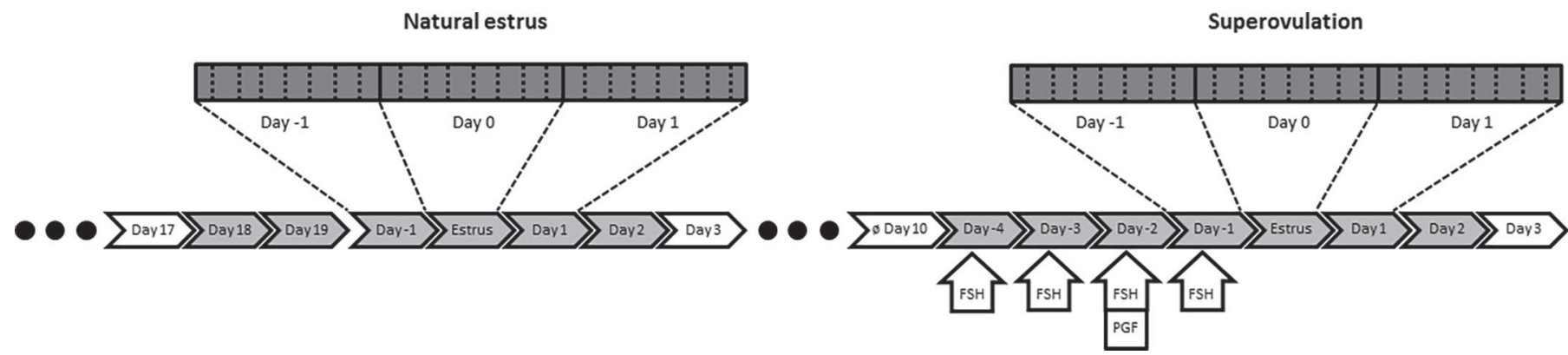

Figure 1. Experimental schedule depicting the overall design and the timing of audiovisual observations, examinations, and blood sampling. The schedule starts from the left with d 17 after previous estrus. The days relative to the day of estrus are shown in arrow boxes; the arrows below indicate the FSH and $\mathrm{PGF}_{2 \alpha}$ administration. Gray highlighted arrow boxes mark the days of examination and sampling. The days and intervals of audiovisual analyses are represented above the arrow boxes (gray bars with dotted lines for intervals).

the postpubertal stage of the animals was confirmed by detecting different follicle stages and a corpus luteum on their ovaries via repeated transrectal ultrasound examinations and at least one estrus during routine visual detection. The estrus before the natural estrus observed in our study was induced by cloprostenol (PGF Veyx forte, Veyx Pharma GmbH, Schwarzenborn Germany). The day of estrus was assessed via visual observation, vaginoscopy, and transrectal ultrasound examinations of the ovaries from the day of cloprostenol administration until ovulation (Determination of the Day of Estrus section). The formation of a corpus luteum was monitored between d 9 and 10 after the cloprostenolinduced estrus. All the heifers in the study had different days of estrus except for 2 cases during NAT, which was due to different sexual cycle lengths, and one case during SUP.

During the experimental period, feed was provided once a day in a TMR ad libitum, and the animals had free access to water. In the mornings (0630 to $0730 \mathrm{~h}$ ), the heifers were fixed in the self-locking feed barrier for the daily cleaning routine, during which examinations and sampling in the peri-estrus period were performed (Examinations and Sampling section).

The experiment was approved by the federal state of Mecklenburg Western-Pomerania (LALLF M-V/ TSD/7221.3-2.1-021/13).

\section{Examinations and Sampling}

Daily vaginoscopy and transrectal ultrasound examination of the ovaries were performed from d 18 after the previous estrus to the day of ovulation for NAT and from the day of the first FSH administration to $2 \mathrm{~d}$ after the last FSH injection for SUP (Figure 1). A tubular speculum was used for vaginoscopy, during which the opening stage and shape of the cervix and the color and degree of moisture of the vaginal mucosa were assessed using the scheme described in Busch and Zerobin (2009). This scheme includes the shape of the portio vaginalis cervicis using 4 categories: peg-shaped, rosette-shaped, broadly shaped, and loose pendently. Furthermore, the opening stage of the cervix was assessed based on 4 categories ranging from completely closed to passable with one finger. The color of the vagina was graded on a 5-level scale from pale to intense redness, and the degree of moisture was classified using 5 levels ranging from dry and sticky to exhibiting fluid accumulation. According to this scheme, the result of a vaginoscopy indicating physiological estrus is a rosette-shaped portio vaginalis cervicis; a pencil can pass through the cervix; and the vagina is hyperemic and intensely moistened.

Within the time periods described for the 2 treatments, ultrasound examinations were performed daily (Figure 1) with a Micromaxx ultrasound system (SonoSite Inc., Bothell, WA) equipped with a linear probe (L $52 \mathrm{x} / 10-5 \mathrm{MHz}$ transducer). During the ultrasound examinations, the diameter of the follicles and the corpus luteum were assessed. Additionally, a blood sample was taken from the coccygeal vein before the ultrasound examination and vaginoscopy and centrifuged at 2,100 $\times g$ at $4^{\circ} \mathrm{C}$ for $10 \mathrm{~min}$. The plasma was transferred into a container and stored at $-20^{\circ} \mathrm{C}$ until analysis.

Visual estrus detection was performed twice a day for at least 20 min through a semi-transparent mirror, and the estrus-related behaviors listed in Table 1 were recorded.

\section{Determination of the Day of Estrus}

For all subsequent analyses, the day of estrus for each heifer was individually identified based on the results of the daily examinations (ultrasound and vaginoscopy) 
and visual estrus detection. The day of estrus was determined when the heifer met all the following criteria: corpus luteum in regression or regressed, a follicle larger than $14 \mathrm{~mm}$ in diameter, and at least a pencil-passable cervix, an intensely moistened vagina and behavioral signs of estrus. During superovulation, the follicle criterion was modified to follicles greater than $10 \mathrm{~mm}$ on both ovaries (in all 12 cases, the criteria were met on the first day after the last FSH administration).

\section{Hormone Analysis}

The plasma concentration of progesterone was measured using a quantitative tritium RIA. The tracer was 1,2,6,7-H(N) progesterone (Hartmann Analytik, Braunschweig, Germany), and the antibody was generated by immunization of rabbits versus 11-OH-progesterone conjugate. For direct progesterone measurement, $50 \mu \mathrm{L}$ of plasma was used, and the incubation time was 30 min at $37^{\circ} \mathrm{C}$ and $2 \mathrm{~h}$ at $4^{\circ} \mathrm{C}$. The intra- and interassay coefficients of variation were calculated as 7.6 and $9.8 \%$, respectively. The analysis followed the protocol previously published by Schneider et al. (2002).

\section{Natural Estrus and Superovulation Treatment}

Natural estrus was defined as the first spontaneously occurring estrus after a previous estrus, and the superovulation treatment was started between $\mathrm{d} 8$ and 14 after this natural estrus. Superovulation was induced by 8 administrations of FSH (Pluset, Laboratorios Calier, S.A., Barcelona, Spain) on 4 consecutive days and 2 injections of cloprostenol (PGF Veyx forte, Veyx Pharma $\mathrm{GmbH}$ ) on the third day of treatment. The injections occurred in the morning and afternoon and were separated by at least $8 \mathrm{~h}$. On the first day of treatment, the heifers received $175 \mathrm{IU}$ of $\mathrm{FSH}$, and the dosage was reduced by $50 \mathrm{IU}$ per d to $25 \mathrm{IU}$ on the fourth day of treatment. Two injections of $0.5 \mathrm{mg}$ of cloprostenol (PGF Veyx forte, Veyx Pharma GmbH) were administered on $\mathrm{d} 3$.

\section{Audiovisual Analysis}

Three days of audiovisual recordings were analyzed for each estrus (day of estrus $\pm 1 \mathrm{~d}$ ). Videos including audio tracks were recorded $24 \mathrm{~h}$ a day on a digital recorder (EDR HD-4H4, EverFocus Electronics Co., New Taipei City, Taiwan) throughout the entire experimental period and stored as 24 -h sections starting at $0000 \mathrm{~h}$ and ending at $0000 \mathrm{~h}$ the following day. The midnight-to-midnight sections were analyzed for each peri-estrus period (day of estrus $\pm 1 \mathrm{~d}$ ) and defined as the number of days relative to estrus $(\mathrm{d}-1,0$, and +1 ). Two cameras (EverFocus HDTV, EverFocus Electronics Co.) were positioned opposite one another to cover the blind spots of each other, and audio tracks were recorded with a microphone (Sennheiser MKE600, frequency range $40 \mathrm{~Hz}$ to $20 \mathrm{kHz}$, Sennheiser electronic GmbH \&Co. KG, Wedemark, Germany) located on the ceiling of the pen.

Video analysis was performed with Observer XT 10 software (Noldus Information Technology BV, Wageningen, the Netherlands), and each estrus-related behavior (Table 1) and vocalization were considered point events. Vocalizations were assigned to a heifer based on visible exhalation or typical head movements (or both) that were synchronous with an audible vocalization on the video (Supplemental File S1; https://doi.org/10 $.3168 /$ jds.2017-13412), and the number of vocalizations for each animal and interval were summed to determine the vocalization rate per interval.

Each 3-d observation period for an individual subject within one estrus type was coded by a single observer, but 2 observers were involved in this study. To ensure inter-observer-reliability, 2 sections of the audiovisual recordings (each approximately $1.2 \mathrm{~h}$ in length) taken during estrus climax were coded by both observers, and Cohen's kappa was $\kappa=0.89$ for frequency of all observed behaviors. Intra-observer reliability was examined by coding and recoding 4 sections of the audiovisual recordings (again, each approximately $1.2 \mathrm{~h}$ in length) by the same observer, and Cohen's kappa was $\kappa$ $=0.91$ for frequency of all observed behaviors.

\section{Determination of Estrus Climax}

To more precisely determine estrus climax, the behavioral data were split into 3 -h intervals, resulting in 8 intervals per day and 24 intervals per estrus type and heifer. Each estrus behavior was summed per interval and multiplied by a factor provided by VanVliet and VanEerdenburg (1996; see Table 1 for definitions and factors), and the sum of all the factorized estrus behaviors per interval was the estrus score. In contrast to the original work, flehmen, mucus discharge, and restlessness were not considered because flehmen and mucus discharge were not reliably detected on video, and restlessness could not be considered a point event. The interval with the highest estrus score was considered the estrus climax and labeled interval 0 (I0).

Additionally, the time interval from estrus climax to ovulation was defined as the middle of I0 until the first ultrasound examination $(0700 \mathrm{~h})$, with the absence of the dominant follicle or a new corpus luteum for NAT and with signs of the first ovulations for SUP. 


\section{Statistical Analyses}

The data were analyzed using the SAS statistical software package version 9.4 for Windows (SAS Institute Inc., Cary, NC). Descriptive statistics and tests for normality were calculated using the UNIVARIATE procedure of Base SAS software.

Progesterone data were analyzed by repeated measures ANOVA using the MIXED procedure with estrus type (NAT, SUP), day $(-4$ to +2$)$, and the estrus type $x$ day interaction as fixed factors. The factors of estrus type and day were repeated factors used with UN@ $\mathrm{AR}(1)$-type blocks for the block diagonal residual covariance matrix in the repeated statement of the MIXED procedure as described above [UN@AR(1) is the direct product of matrices UN and AR(1), where UN denotes the unstructured type of the covariance matrix for the factor of estrus type and $\mathrm{AR}(1)$ denotes the first-order autoregressive covariance structure of the covariance matrix for the factor of day].

The time of day (or, to be more precise, the 3-h interval) with the highest estrus score (I0) differed between subjects, leading to different numbers of intervals before and after I0. To compare an equal number of intervals per heifer and estrus, only the intervals from I-9 to I+9 were considered in the statistical analyses. Estrus behavior, estrus score, and vocalization data were analyzed by repeated measures ANOVA with the MIXED procedure, and the ANOVA models contained the fixed factors of estrus type (levels: NAT and SUP), interval (I-9 to I+9), and the estrus type $\times$ interval interaction. The factors of estrus type and interval were repeated factors used with UN@AR(1)-type blocks for the block diagonal residual covariance matrix in the repeated statement of the MIXED procedure.

Least squares means and their standard errors were computed for each fixed effect in the models, and all the pairwise differences were tested by the Tukey-Kramer test, a procedure for multiple pairwise comparisons. Additionally, the SLICE statement of the MIXED procedure was used to perform partitioned analyses of the LSM for the 2-way interaction of estrus type $\times$ interval (estrus type $\times$ day); that is, test of the estrus types within the levels of interval (or day) and test of the intervals (or day) within the levels of estrus type.

\section{RESULTS}

All 12 heifers ovulated during both natural estrus and superovulation, so data from 12 natural estruses and 12 superovulations were included in the analyses.

\section{Examinations}

Natural estrus occurred an average $( \pm \mathrm{SD})$ of 22.6 $( \pm 2.9) \mathrm{d}$ after the previous estrus. In SUP, the first FSH injection was administered on d $11( \pm 1.3)$, on average, after estrus, and the subsequent estrus was detected on the day after the last FSH injection in all 12 subjects.

As expected, vaginoscopy showed vaginal mucus accumulation and an opened cervix on the day of estrus. The average time interval from estrus climax to ovulation was $39.1 \mathrm{~h}( \pm 2.3)$ in NAT and $30.5 \mathrm{~h}( \pm 3.1)$ in SUP. On the day of ovulation, as detected by transrectal ultrasonography, the degree of mucus in the vaginoscopy differed among heifers, ranging from fluid accumulation to moderate humidity, and the stages of the opening of the cervix decreased or remained constant.

\section{Hormone Analysis}

For both estrus types, the serum progesterone concentration was influenced by the fixed factor of day $\left(\mathrm{F}_{6,45.5}=23.17 ; P<0.001\right)$; it reached the minimum on the day of estrus in both NAT and SUP, decreasing below $1 \mathrm{ng} / \mathrm{mL}$ (NAT: $0.41 \pm 0.28 \mathrm{ng} / \mathrm{mL}$; SUP: 0.58 $\pm 0.29 \mathrm{ng} / \mathrm{mL}$ ). The serum progesterone levels in NAT and SUP on $\mathrm{d}-4,-3$, and -2 were significantly higher than those on $\mathrm{d}-1,0,+1$, and $+2($ all $|\mathrm{t}|>5.03 ; P<$ 0.001).

Progesterone concentration was also influenced by estrus type $\left(\mathrm{F}_{1,22.2}=13.9 ; P=0.001\right)$. Serum concentrations were significantly higher with SUP than NAT on $\mathrm{d}-4\left(\mathrm{t}_{61.4}=2.02 ; P=0.047\right),-3\left(\mathrm{t}_{51.1}=3.36 ; P\right.$ $=0.001),-2\left(\mathrm{t}_{48.0}=4.02 ; P<0.001\right)$, and $+2\left(\mathrm{t}_{51.8}=\right.$ $2.54 ; P=0.014$; Figure 2).

\section{Behavior Analysis}

The statistical analysis of the fixed factors of interval and estrus type and their interaction for the 6 observed estrus behaviors revealed the following: the interval affected 4 of the 6 estrus behaviors [standing $\left(\mathrm{F}_{18,132.7}=\right.$ 8.32; $P<0.001)$, mounting head $\left(\mathrm{F}_{18,131.3}=2.36 ; P=\right.$ $0.003)$, mounting $\left(\mathrm{F}_{18,173.2}=4.14 ; P<0.001\right)$, and chin rest $\left.\left(\mathrm{F}_{18,271.6}=14.91 ; P<0.001\right)\right]$. Estrus type alone did not influence any of the estrus behaviors, but there were significant effects of the estrus type $\times$ interval interaction on mounted run $\left(\mathrm{F}_{18,259.4}=1.69 ; P=0.041\right)$, mounting head $\left(\mathrm{F}_{18.304 .1}=1.89 ; P=0.016\right)$, and chin rest $\left(\mathrm{F}_{18,402.0}=3.39 ; P<0.001\right)$.

Pairwise comparisons of the intervals between the 2 estrus types revealed the following differences in be- 
havior between NAT and SUP for the given intervals (Figure 3): During NAT, mounted run was more frequent in $\mathrm{I}-4\left(\mathrm{t}_{214.7}=2.76 ; P=0.006\right)$; standing was more frequent in I-2 $\left(\mathrm{I}-2, \mathrm{t}_{234.3}=1.97 ; P=0.005\right)$, I0 $\left(\mathrm{t}_{234.3}=2.96 ; P=0.003\right)$, and $\mathrm{I}+1\left(\mathrm{t}_{234.3}=2.77 ; P=\right.$ $0.006)$; mounting occurred more frequently in $\mathrm{I}-2\left(\mathrm{t}_{200.8}\right.$ $=2.89 ; P=0.004)$; mounting head was more frequent during $\mathrm{I}+2\left(\mathrm{t}_{237.0}=2.73 ; P=0.007\right)$; and contrary to the mounting-related behaviors, chin rest was less frequent in NAT compared with SUP in intervals I0 $\left(\mathrm{t}_{302.0}=3.66 ; P<0.001\right), \mathrm{I}+1\left(\mathrm{t}_{302.0}=3.28 ; P=0.001\right)$, and $\mathrm{I}+2\left(\mathrm{t}_{302.0}=2.43 ; P=0.016\right)$.

\section{Estrus Score and Vocalization}

Estrus score was significantly affected by interval $\left(\mathrm{F}_{18,160.6}=19.85 ; P<0.001\right)$ but not by estrus type $\left(\mathrm{F}_{1,70.6}=0.05 ; P=0.83\right)$ or the estrus type $\times$ interval interaction $\left(\mathrm{F}_{18,266.3}=0.92 ; P=0.56\right)$. Comparing estrus types by interval, a higher estrus score was observed in NAT at $\mathrm{I}-2\left(\mathrm{t}_{195.4}=2.11 ; P=0.035\right)$, but no significant differences were detected between estrus types in the other intervals.

In consecutive intervals (Figure 4), a significant increase in estrus score was first detected between I-3

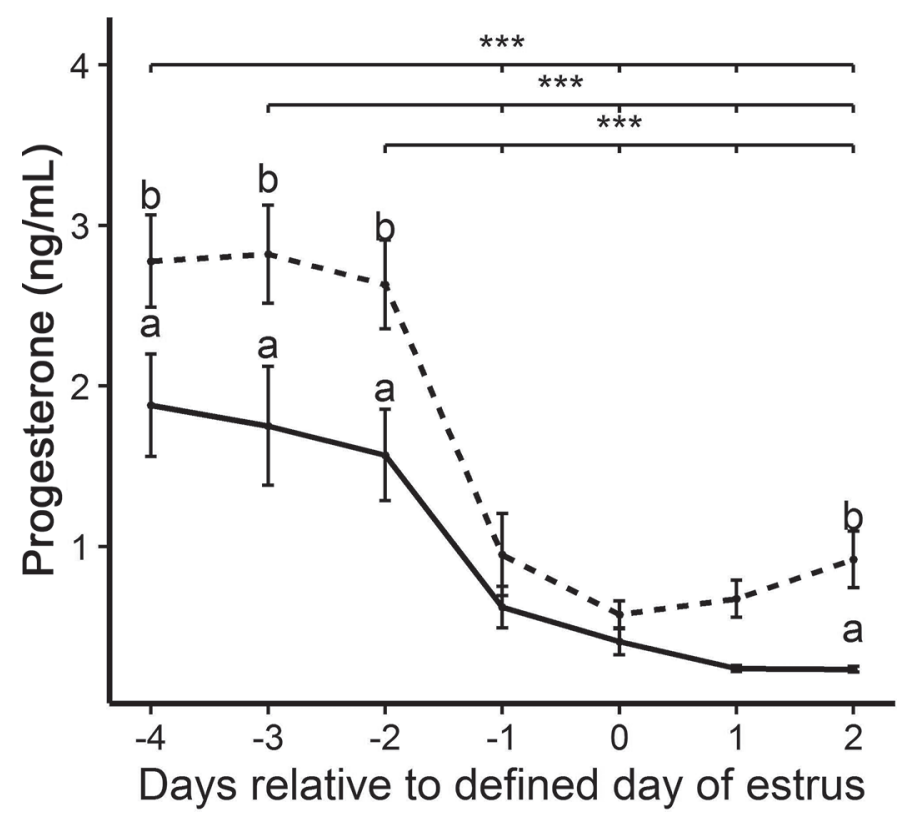

Figure 2. Plasma concentration of progesterone for natural estrus (solid line) and for superovulation treatment (dashed line). Error bars mark the SE in both estrus types. Different letters (a,b) indicate significant differences between the treatments on the specific days. Horizontal lines mark significant differences between the days for the 2 types of estrus $(* * * P<0.001)$. and $\mathrm{I}-2\left(\mathrm{t}_{248.4}=4.59 ; P=0.001\right)$ for NAT followed by a second, significant increase from $\mathrm{I}-1$ to $\mathrm{I} 0\left(\mathrm{t}_{248.4}=\right.$ 7.92; $P<0.001)$ and a subsequent decrease from I0 to $\mathrm{I}+1\left(\mathrm{t}_{248.4}=9.24 ; P<0.001\right)$. For SUP, a significant increase in estrus score points was observed between I-2 and I-1 that continued to I0 (I-2: $\mathrm{t}_{189.0}=5.18$; $\left.P<0.001 ; \mathrm{I}-1: \mathrm{t}_{189.0}=8.08 ; P<0.001\right)$ followed by a significant decrease between I0 and I+1 $\left(\mathrm{t}_{189.0}=3.62\right.$; $P=0.041$ ).

Comparing estrus scores between intervals, a special focus was placed on intervals $I-1, I 0$, and $I+1$ because these intervals are close to the estrus climax and therefore most relevant to the aim of the study. These results are described below, first for NAT and then for SUP.

In NAT, the I0 estrus score was significantly higher than that of all other intervals (all $|\mathrm{t}|>7.87 ; P<$ 0.001 ). In interval I-1, the estrus score was already significantly higher than that in all intervals from I-9 to I-3 (all $|\mathrm{t}|>5.3 ; P<0.001$ ) and from $\mathrm{I}+3$ to $\mathrm{I}+9$ (all $|\mathrm{t}|>4.89 ; P<0.001)$. I+1 estrus scores remained higher than scores in I-9 to I-4 (all $|\mathrm{t}|>4.59 ; P<$ 0.01 ) and $\mathrm{I}+3$ to $\mathrm{I}+9$ (all $|\mathrm{t}|>4.87 ; P<0.001$ ).

In SUP, the estrus score pattern was similar to the estrus score progression in NAT; the estrus score was significantly higher in I0 (all $|\mathrm{t}|>8.08 ; P<0.001)$ than in all other intervals. Compared with I-1, we found significantly higher estrus scores in the intervals I-9 to I-2 (all $|\mathrm{t}|>4.60 ; P<0.001)$ and $\mathrm{I}+3$ to $\mathrm{I}+9(|\mathrm{t}|>$ 4.27; $P<0.01)$, and the score at $\mathrm{I}+1$ was significantly higher than those for intervals $\mathrm{I}-9$ to $\mathrm{I}-4$ (all $|\mathrm{t}|>$ 4.14; $P<0.01)$ and intervals $\mathrm{I}+2$ to $\mathrm{I}+9($ all $|\mathrm{t}|>3.61$; $P<0.05)$.

The vocalization rates varied within and among heifers, ranging from 0 in one heifer to 489 vocalizations per estrus. During the peri-estrus period, a total of 1,529 vocalizations were observed in NAT and 773 vocalizations in SUP. Comparing the vocalizations on a day-to-day basis, the mean vocalizations $( \pm \mathrm{SE})$ were $9.6( \pm 3.8)$ in NAT and $24( \pm 8.3)$ in SUP per day for $\mathrm{d}$ $-1,110.3( \pm 41.2)$ during NAT and $33.4( \pm 19.1)$ during SUP per day on $\mathrm{d} 0$, and $7.5( \pm 2.8)$ in NAT and 7 $( \pm 3.5)$ in SUP per day on $d+1$. The vocalization rate during estrus climax (I0) was $27.58( \pm 9.76)$ for NAT and $16.17( \pm 11.6)$ for SUP, and the highest average vocalization rate was detected in I-1 (42.58 \pm 21.89$)$ for NAT and in I0 for SUP. The vocalization rate was affected by interval $\left(\mathrm{F}_{18,222.4}=2.90 ; P<0.001\right)$, but estrus type $\left(\mathrm{F}_{1,88.0}=1.52 ; P=0.22\right)$ and the estrus type $\times$ interval interaction $\left(\mathrm{F}_{18,264.9}=1.52 ; P=0.081\right)$ had no significant effect.

Comparing consecutive intervals, a significant increase in vocalization rate was only detected between $\mathrm{I}-2$ and $\mathrm{I}-1\left(\mathrm{t}_{266.5}=4.34 ; P=0.003\right)$ in NAT; no sig- 


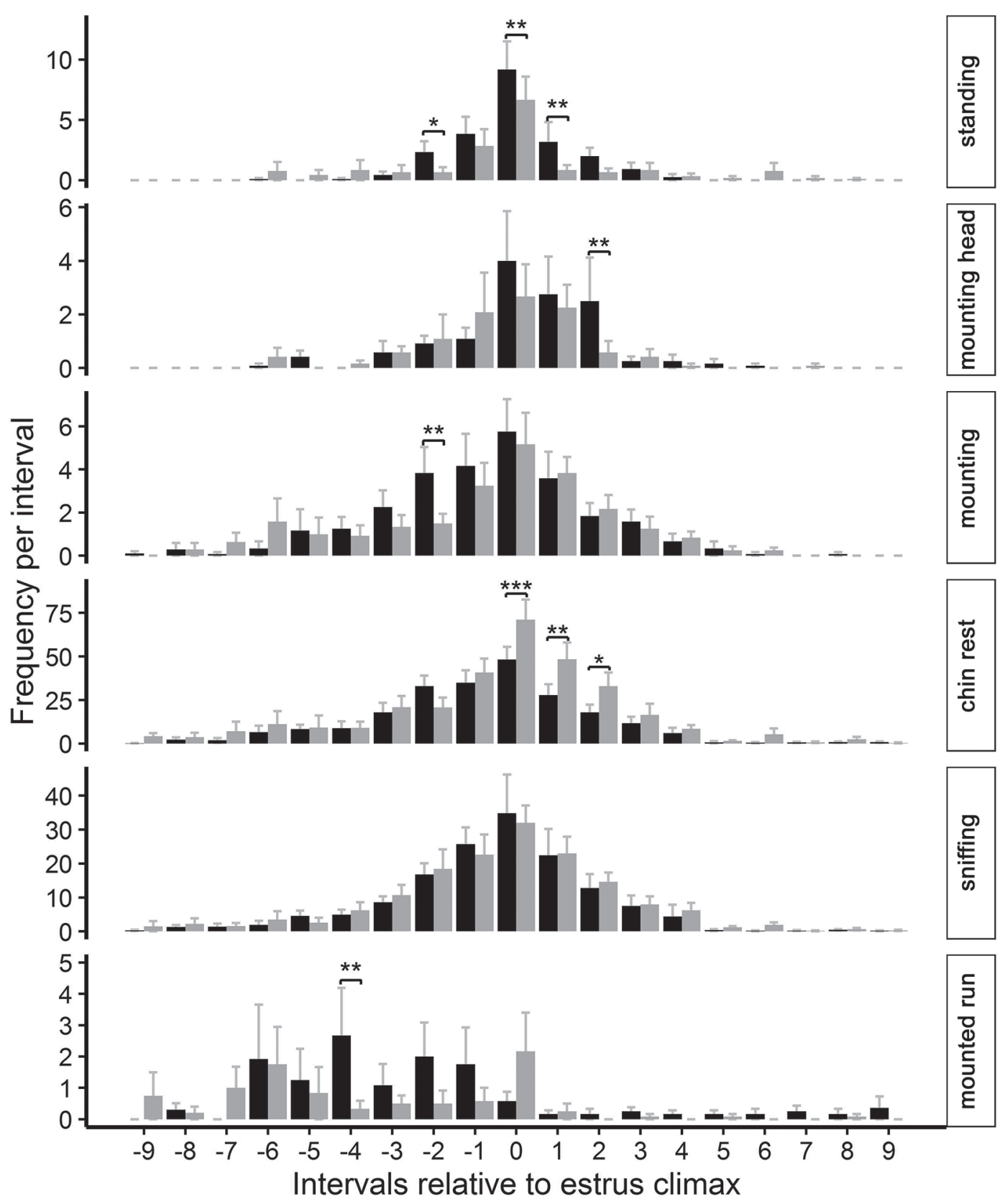

Figure 3. Mean number of the 6 observed behaviors per 3-h interval. The black bars represent natural estrus, and the gray bars represent superovulation treatment. Error bars indicate the SE. The horizontal lines indicate significant differences between estrus types within an interval $\left(* P<0.05,{ }^{* *} P<0.01,{ }^{* * *} P<0.001\right)$.

nificant increase in consecutive intervals was detected in SUP (Figure 4).

Comparing the vocalization rates per interval between estrus types, a significantly higher rate of vocalizations was identified at $\mathrm{I}-1\left(\mathrm{t}_{226.6}=4.87 ; P<0.001\right)$ in NAT. Additionally, a tendency of higher vocalization rates in NAT than in SUP was observed at $\mathrm{I}-2\left(\mathrm{t}_{226.6}=1.91 ; P\right.$ $=0.058)$ and $\mathrm{I} 0\left(\mathrm{t}_{226.6}=1.79 ; P=0.075\right)$.
For NAT, the vocalization rate was significantly higher at I-1 than I-9 to I-2 (all $|\mathrm{t}|>4.34 ; P<0.01)$ and $\mathrm{I}+1$ to $\mathrm{I}+9($ all t $>4.38 ; P<0.01)$.

For SUP, the vocalization rates at I0 and I-1 were not significantly different. The vocalization rate in SUP was not significantly different between intervals. However, a tendency was observed (all t $<3.33 ; P<0.1$ ) for intervals $\mathrm{I}-7, \mathrm{I}-6, \mathrm{I}-4, \mathrm{I}+2, \mathrm{I}+7, \mathrm{I}+8$, and $\mathrm{I}+9$ 
compared with $\mathrm{I} 0$, which showed the highest vocalization rate.

\section{DISCUSSION}

In our study, the vocalization rates of dairy heifers increased in the 3-h interval before estrus behavior climax, but this increase was less pronounced and only a statistical tendency under superovulation treatment than in natural estrus. During natural estrus, the vocalization rate significantly increased close to the climax of estrus behavior.

The significant increase in vocalization in our study was consistent with the results of the previous studies

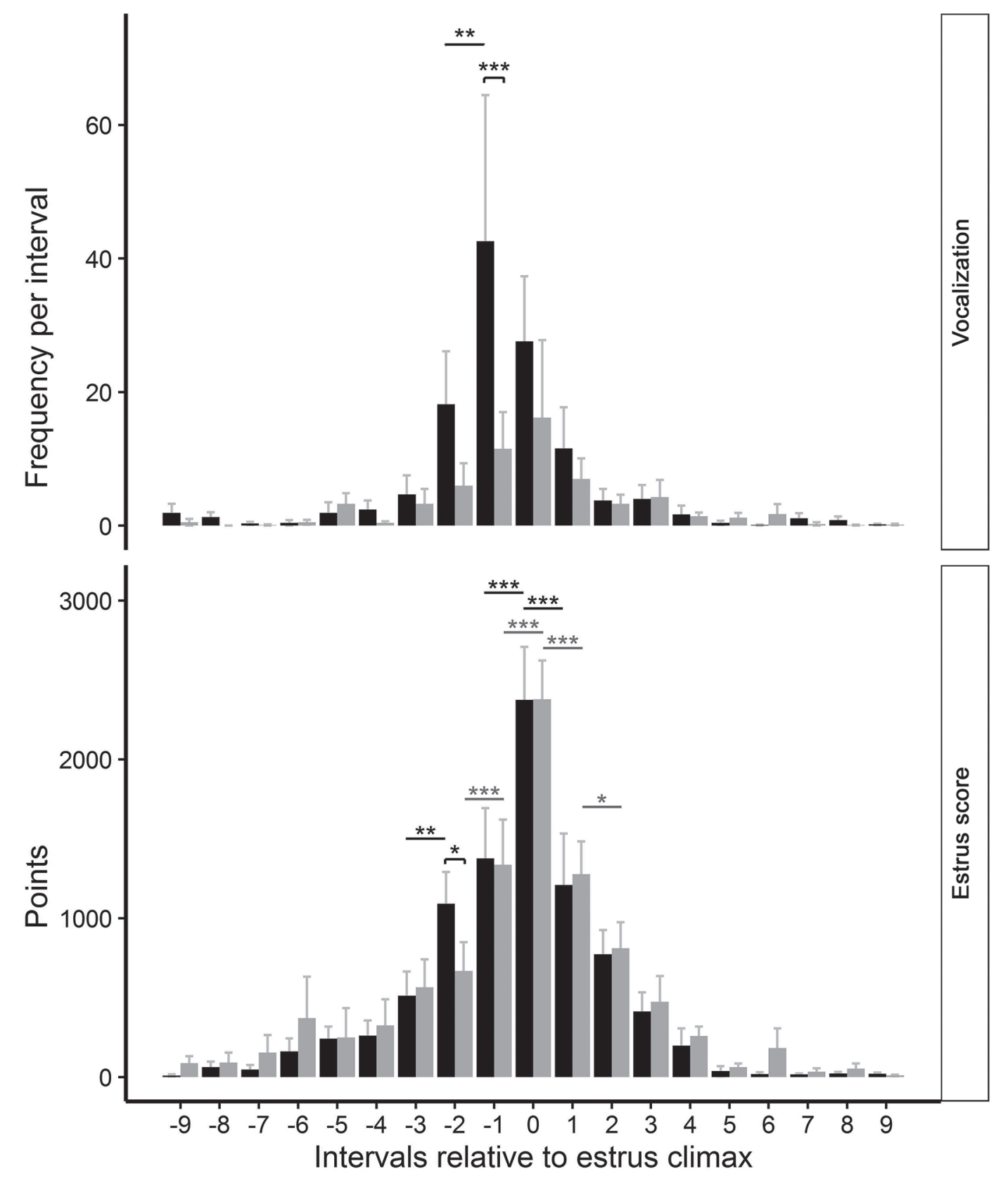

Figure 4. The upper graph shows the average vocalization rate per 3-h interval separately for natural estrus in black bars and superovulation treatment in gray bars. The bottom graph shows the calculated estrus score per interval with the identical color code. Error bars indicate the SE. Small horizontal brackets indicate significant differences between the estrus types within the interval. Long (black = natural estrus and gray $=$ superovulation) horizontal lines indicate significant differences in consecutive intervals within natural estrus or superovulation $\left({ }^{*} P<\right.$ $\left.0.05,{ }^{* *} P<0.01,{ }^{* * *} P<0.001\right)$. 
of Schön et al. (2007) and Dreschel et al. (2014), so we could confirm the increase in vocalization toward estrus first found in tiestall-housed and then in loose-housed dairy heifers during both natural and cloprostenolinduced estrus. On the one hand, that the pattern of vocal activity seems to be consistent across different housing systems and hormonal fertility treatments indicates its biological significance, and on the other hand, it supports its future use as an additional indicator in automated estrus detection devices.

It is crucial for dairy farmers to know when to inseminate a cow. Therefore, it is important to gain knowledge about the temporal relationship between ovulation and potential behavioral estrus indicators that could then be used for estrus detection. A study conducted by Walker et al. (1996) revealed an average time from the first standing-to-be-mounted event to ovulation of 27.6 $\mathrm{h}( \pm 5.4)$, which was confirmed by Aungier et al. (2015). The estradiol peak has been previously shown to be synchronous to estrus score climax (Lyimo et al., 2000), and it precedes ovulation by $35 \mathrm{~h}$ (Aungier et al., 2015). To determine the timeframe of ovulation after the peak vocalization rate, we used the same estrus score as Lyimo et al. (2000). In NAT, ovulation was detected $39.1 \mathrm{~h}$ after estrus climax while the vocalization peak was in interval I0 (i.e., $3 \mathrm{~h}$ earlier), which resulted in an average time interval of $42.1 \mathrm{~h}$ from vocalization peak to ovulation in NAT. In SUP, ovulation was detected $30.5 \mathrm{~h}$ after estrus climax, which coincided with the vocalization peak, so the average time interval from vocalization peak to ovulation was $30.5 \mathrm{~h}$. The higher values for NAT in our study compared with those of Aungier et al. (2015) might possibly the result of the less frequent ovulation controls. The shorter time to first ovulation during SUP reflects multiple ovulations, and the period of ovulations is consistent with Kanitz et al. (2002).

Lyimo et al. (2000) showed that while estrus scores and estradiol peaked simultaneously, commercially available automated estrus detection devices identified activity clusters only 6 to $12 \mathrm{~h}$ after the estradiol peak. This time frame is consistent with Aungier et al. (2015), who reported activity clusters $8 \mathrm{~h}$ after the estradiol peak (i.e., 29 to 23 and $27 \mathrm{~h}$ before ovulation based on these 2 studies). Given that our data set showed that the peak vocalization rate preceded ovulation by $42.1 \mathrm{~h}$ (NAT) and $30.5 \mathrm{~h}$ (SUP), vocalization rate detection presents a time advantage compared with activity measurement. These results highlight that vocalization has the potential to enhance estrus detection. However, this study only considers the behavior of heifers, so further studies are needed to investigate the vocal behavior of lactating dairy cows. If applied to AI, vocalization analysis allows trained technicians/farmers or veterinarians to obtain information about cows in estrus even earlier than with activity clusters alone. Therefore, we suggest implementing vocalization rate detectors in automated estrus detectors, which would require that the vocalization of individual animals in loose housed groups be automatically recorded and correctly assigned as well as the development of algorithms to identify peaks in vocalization rates. However, before an on-farm solution can be developed, several technical advances are required, especially concerning the reliable detection of vocalizations and correct attribution to the calling individual. A ready-to-use on-farm solution should operate without requiring either a data set of individual, good-quality vocalization recordings to track cows based on individual vocal characteristics (Yajuvendra et al., 2013) or time-consuming video analysis. Additionally, acoustic triangulation involving the use of positioning systems is difficult because the constant ambient noises interfere with this method as well as the limited accuracy of positioning systems. Hence, there is a need for new technical solutions to automatically record and analyze vocalizations by freely moving animals and attribute them to the individual caller with high sensitivity and specificity but without the need for labor-intensive procedures. With such a device integrating vocalization and activity measures, dairy farmers could time inseminations before detecting activity clusters. Because AI depends on experienced personnel, it is beneficial for farmers to have an extended planning period to ensure that the necessary laborer is present for the best results.

According to the literature, little is known about the vocal contribution of cows to mating behavior, but as mentioned above, the time course of vocalization during the sexual cycle seems to be quite consistent in dairy heifers across housing systems and hormonal treatments and is thus likely to be of biological significance. In some species, altered vocalization behavior occurs shortly before the receptive period. Matochik et al. (1992) reported higher rates of ultrasonic vocalizations in the proestrus and early estrus period of Long-Evans rats, and in African elephants, Leong et al. (2003) observed an increase in low-frequency vocalizations during the nonovulatory follicular phase. In a replay experiment, where calls of female giant pandas were played to males, Charlton et al. (2010) showed that male giant pandas react differently to the calls of females in the pre-fertile phase compared those in the fertile phase. These findings suggest a vocal contribution by females to mating behavior, which might not be as obvious as male vocal behavior in some species [e.g., red deer during mating season (Bocci et al., 2013)]. An 
increase in the vocalization rate of cattle may function as a signal for male mating partners within listening range to approach, but whether these calls differ from vocalizations during diestrus or only serve as passive information obtained by potential mating partners by eavesdropping (McGregor, 1993; Leong et al., 2003) is not yet well understood. Another possible explanation for high vocalization rates in cattle during estrus is that cattle are more vocal under stressful [e.g., handling (Grandin, 2001)] and painful situations [e.g., branding (Lay et al., 1992; Stookey et al., 1996)]. These situations result in the release of increased amounts of catecholamine and cortisol (Lay et al., 1992), and Lyimo et al. (2000) found a temporal coherence between the climax of estrus behavior and a slight increase in cortisol plasma levels. Therefore, the physiological increase in cortisol in estrus and the heightened arousal level of animals might also be a possible reason for an increased vocalization rate during estrus.

In a second step of this study, the effects of natural estrus and superovulation on estrus behavior and vocalization were compared. The overall vocalization rate was lower during SUP than in NAT, but the rate was only significantly different between NAT and SUP in I-1. The difference in vocalization might occur because of different stages in the development of multiple ovarian follicles during superovulation. During the daily control of ovulation, first ovulations were detected earlier in heifers subjected to superovulation treatment than in those in natural estrus, and in most cases, the ovulation control on the following day revealed more ovulations than the day before. This is also described in a study by Kanitz et al. (2002), who observed an ovulation period of up to $12 \mathrm{~h}$ in superovulated cows and heifers. This indicates that some follicles become ovulation competent before other follicles within the same animal, and because of the different stages of development, the pattern of steroid hormone secretion in these follicles most likely differs from that of the single follicle situation during natural estrus.

The different stages in follicular development were also mirrored in the progesterone plasma concentrations. In our study, significantly higher progesterone plasma concentrations before estrus were measured during SUP than in NAT, which reflected different phases in the sexual cycle. As expected, animals undergoing superovulation had a higher progesterone plasma concentration before estrus $(\mathrm{d}-4$ to -2$)$ than the animals during natural estrus with a corpus luteum in regression. The higher progesterone levels at $d+2$ in SUP reflected earlier and multiple ovulations induced by superovulation.
Hormones, particularly the follicle-produced estradiol, likely affect estrus behavior. For example, Lyimo et al. (2000) found a positive correlation between the maximum estradiol level and the total estrus score, and Roberge et al. (1995) found that superovulated Holstein heifers had high amounts of estradiol in the plasma. To investigate the effect of superovulation and thus the possible effect of higher estradiol production on estrus behavior traits, Jezierski (1993) conducted a study with black and white dairy cows using a similar superovulation protocol, and he reported no significant changes in mounting and received mountings but found a higher frequency of initiated and received sniffings in the superovulated animals compared with nonsuperovulation estruses. We could not confirm these sniffing behavior findings with SUP, but we did not account for received sniffings. In another study by Jimenez et al. (2011), synchronized and superovulated Bos indicus cattle were compared using a superovulation treatment similar to that in our study, and they found a 50\% increase in mountings per hour in addition to higher counts of mountings, mounting attempts, head bumping, flehmen, sniffing, following, and licking in the superovulated animals. In our heifers, the 4 analyzed mounting behaviors (standing, mounted run, mounting head, and mounting) were all observed in at least one interval, with significantly more mounting behaviors in natural estrus than in superovulation. Particularly, more standing-to-be-mounted occurred during NAT in 3 intervals $(\mathrm{I}-2, \mathrm{I} 0, \mathrm{I}+1)$. However, all observed mounting behaviors (standing, mounting head, mounting, mounted run) were observed in low numbers per interval, so slight differences triggered by other factors (e.g., seasonal and intra-individual variations in mounting behaviors) might have had an effect. Coe and Allrich (1989) also reported very large variations in different mounting behaviors in both nonsuperovulation and superovulation periods. The significant increase in the estrus score at I-2 for NAT in our study was likely due to more active mountings and standing-to-be-mounted events in that interval because these 2 behaviors greatly affect the estrus score (standing equals 100 points and mounting equals 35 points per event).

The administration of FSH and, therefore, the increase in the number of growing follicles might not be the sole explanation for the observed differences between NAT and SUP because the number and characteristics of estrus behavior traits can differ at relevant levels within animals (Rottensten and Touchberry, 1957). Comparing the estrus scores, the 2 types of estrus only differed in one interval, so although some differences were observed in certain estrus behaviors, the course 
of the estrus score remained similar between the estrus types.

\section{CONCLUSIONS}

To our knowledge, the similar temporal course between vocalization rate and estrus behavior has not been previously reported. During natural estrus, we demonstrated a clear relationship between the climax of estrus behavior and the peak of vocalization. This relationship was less pronounced during superovulation, although vocalization tended to peak directly at the climax of estrus behavior. The temporal association of vocalization rate and estrus behavior highlights the potential of vocalization as parameter in the detection of estrus. To exploit this potential, we need to overcome the current challenges of detecting individual vocalization in group-housed dairy cattle. Implementing vocalization as component of automatic estrus detection devices could be beneficial for the determination of the climax of estrus behavior.

\section{ACKNOWLEDGMENTS}

We thank the staff of the Experimental Facility for Cattle of the Leibniz Institute for Farm Animal Biology and our technicians of the Institute of Behavioural Physiology and Institute of Reproductive Biology for their excellent support during and after the experiments. Special thanks are extended to Katrin Siebert and Kurt Wendland from the Institute of Behavioural Physiology for their technical and social support. The European Social Fund Mecklenburg-Western Pomerania (V-630-F-124-2011/150 V-630-S-124-2011/151) supported this work.

\section{REFERENCES}

Aungier, S. P. M., J. F. Roche, P. Duffy, S. Scully, and M. A. Crowe. 2015. The relationship between activity clusters detected by an automatic activity monitor and endocrine changes during the periestrous period in lactating dairy cows. J. Dairy Sci. 98:1666-1684. https://doi.org/10.3168/jds.2013-7405.

Bocci, A., M. Telford, and P. Laiolo. 2013. Determinants of the acoustic behaviour of red deer during breeding in a wild alpine population, and implications for species survey. Ethol. Ecol. Evol. 25:5269. https://doi.org/10.1080/03949370.2012.705331.

Bryant, G. A., and M. G. Haselton. 2009. Vocal cues of ovulation in human females. Biol. Lett. 5:12-15. https://doi.org/10.1098/rsbl 2008.0507

Busch, W., and K. Zerobin. 2009. Methoden Der Gynäkologischen Zuchthygienischen Untersuchung. Pages 83-89 in Fruchtbarkeitskontrolle bei Groß- und Kleintieren. W. Busch and K. Zerobin, ed. Studien Sonderausgabe der 1. Auflage. Enke Verlag, Stuttgart, Germany.

Charlton, B. D., J. L. Keating, R. G. Li, H. Yan, and R. R. Swaisgood. 2010. Female giant panda (Ailuropoda melanoleuca) chirps advertise the caller's fertile phase. Proc. Biol. Sci. 277:1101-1106. https://doi.org/10.1098/rspb.2009.1431.

Coe, B. L., and R. D. Allrich. 1989. Relationship between endogenous estradiol-17-beta and estrous behavior in heifers. J. Anim. Sci. 67:1546-1551.

Dreschel, S., P. C. Schon, W. Kanitz, and E. Mohr. 2014. Vocalization of dairy cattle during the oestrous cycle in two different housing systems. Zuchtungskunde 86:157-169.

Firk, R., E. Stamer, W. Junge, and J. Krieter. 2002. Automation of oestrus detection in dairy cows: A review. Livest. Prod. Sci. 75:219-232. https://doi.org/10.1016/S0301-6226(01)00323-2.

Grandin, T. 2001. Cattle vocalizations are associated with handling and equipment problems at beef slaughter plants. Appl. Anim. Behav. Sci. 71:191-201. https://doi.org/10.1016/S0168-1591(00)00179-9.

Ikeda, Y., and Y. Ishii. 2008. Recognition of two psychological conditions of a single cow by her voice. Comput. Electron. Agric. 62:67-72. https://doi.org/10.1016/j.compag.2007.08.012.

Jahns, G. 2008. Call recognition to identify cow conditions-A callrecogniser translating calls to text. Comput. Electron. Agric. 62:54-58. https://doi.org/10.1016/j.compag.2007.09.005.

Jezierski, T. 1993. The Effects of superovulation hormone treatments on the frequency of estrous behaviors in cows. Appl. Anim. Behav. Sci. 36:81-86. https://doi.org/10.1016/0168-1591(93)90100-4.

Jimenez, A., F. Bautista, C. Galina, J. Romero, and I. Rubio. 2011. Behavioral characteristics of Bos indicus cattle after a superovulatory treatment compared to cows synchronized for estrus. AsianAustralas. J. Anim. Sci. 24:1365-1371. https://doi.org/10.5713/ ajas.2011.11032.

Kanitz, W., F. Becker, F. Schneider, E. Kanitz, C. Leiding, and R. Pohland. 2002. Superovulation in cattle: Practical aspects of gonadotropin treatment and insemination. Reprod. Nutr. Dev. 42:587-599. https://doi.org/10.1051/rnd:2002045.

Langmore, N. E., and N. B. Davies. 1997. Female dunnocks use vocalizations to compete for males. Anim. Behav. 53:881-890. https:// doi.org/10.1006/anbe.1996.0306.

Langmore, N. E., N. B. Davies, B. J. Hatchwell, and I. R. Hartley. 1996. Female song attracts males in the alpine accentor Prunella collaris. Proc. Biol. Sci. 263:141-146. https://doi.org/10.1098/ rspb.1996.0022.

Lay, D. C., T. H. Friend, K. K. Grissom, C. L. Bowers, and M. E. Mal. 1992. Effects of freeze or hot-iron branding of angus calves on some physiological and behavioral indicators of stress. Appl. Anim. Behav. Sci. 33:137-147.

Leong, K. M., A. Ortolani, L. H. Graham, and A. Savage. 2003. The use of low-frequency vocalizations in African elephant (Loxodonta africana) reproductive strategies. Horm. Behav. 43:433-443. https://doi.org/10.1016/S0018-506X(03)00025-4.

Lyimo, Z. C., M. Nielen, W. Ouweltjes, T. A. M. Kruip, and F. J. C. M. van Eerdenburg. 2000. Relationship among estradiol, cortisol and intensity of estrous behavior in dairy cattle. Theriogenology 53:1783-1795. https://doi.org/10.1016/S0093-691X(00)00314-9.

Matochik, J. A., N. R. White, and R. J. Barfield. 1992. Variations in scent marking and ultrasonic vocalizations by Long-Evans rats across the estrous cycle. Physiol. Behav. 51:783-786. https://doi .org/10.1016/0031-9384(92)90116-J.

McGregor, P. K. 1993. Signaling in territorial systems-A context for individual identification, ranging and eavesdropping. Proc. R. Soc. Lond. B Biol. Sci. 340:237-244. https://doi.org/10.1098/rstb.1993 .0063 .

Peralta, O. A., R. E. Pearson, and R. L. Nebel. 2005. Comparison of three estrus detection systems during summer in a large commercial dairy herd. Anim. Reprod. Sci. 87:59-72. https://doi.org/10 $.1016 /$ j.anireprosci.2004.10.003

Rae, D. O., P. J. Chenoweth, M. A. Giangreco, P. W. Dixon, and F. L. Bennett. 1999. Assessment of estrus detection by visual observation and electronic detection methods and characterization of factors associated with estrus and pregnancy in beef heifers. Theriogenology 51:1121-1132. https://doi.org/10.1016/S0093 -691X(99)00056-4. 
Reith, S., and S. Hoy. 2012. Relationship between daily rumination time and estrus of dairy cows. J. Dairy Sci. 95:6416-6420. https:// doi.org/10.3168/jds.2012-5316.

Roberge, S., D. Rieger, and N. C. Rawlings. 1995. Periovulatory LH FSH and steroid-hormone profiles in superovulated and unstimulated Holstein heifers. Theriogenology 44:59-70. https://doi.org/ 10.1016/0093-691X(95)00148-2.

Rorie, R. W., T. R. Bilby, and T. D. Lester. 2002. Application of electronic estrus detection technologies to reproductive management of cattle. Theriogenology 57:137-148. https://doi.org/10.1016/ S0093-691X(01)00663-X.

Rottensten, K., and R. W. Touchberry. 1957. Observations on the degree of expression of estrus in cattle. J. Dairy Sci. 40:1457-1465.

Rutten, C. J., W. Steeneveld, C. Inchaisri, and H. Hogeveen. 2014. An ex ante analysis on the use of activity meters for automated estrus detection: To invest or not to invest? J. Dairy Sci. 97:6869-6887. https://doi.org/10.3168/jds.2014-7948.

Schneider, F., A. Bellmann, F. Becker, S. B. Poernomo, C. Rehfeldt G. Nurnberg, and W. Kanitz. 2002. Gonadotropin release in periovulatory heifers after $\mathrm{GnRH}$ analogs measured by two types of immunoassays. Exp. Clin. Endocrinol. Diabetes 110:235-244. https://doi.org/10.1055/s-2002-33073.

Schön,, P., K. Haemel, B. Puppe, A. Tuchscherer, W. Kanitz, and G. Manteuffel. 2007. Altered vocalization rate during the estrous cycle in dairy cattle. J. Dairy Sci. 90:202-206. https://doi.org/10 .3168/jds.S0022-0302(07)72621-8.

Stookey, J., J. Watts, and K. Schwartzkopf. 1996. Effects of restraint and branding on subsequent ease of movement through a chute in beef cattle. J. Anim. Sci. 74(Suppl. 1):133. (Abstr.)

Tobias, M. L., S. S. Viswanathan, and D. B. Kelley. 1998. Rapping, a female receptive call, initiates male-female duets in the South African clawed frog. Proc. Natl. Acad. Sci. USA 95:1870-1875. https://doi.org/10.1073/pnas.95.4.1870.

VanVliet, J. H., and F. J. C. M. VanEerdenburg. 1996. Sexual activities and oestrus detection in lactating Holstein cows. Appl. Anim. Behav. Sci. 50:57-69. https://doi.org/10.1016/0168-1591(96)01068 -4 .

Walker, W. L., R. L. Nebel, and M. L. McGilliard. 1996. Time of ovulation relative to mounting activity in dairy cattle. J. Dairy Sci. 79:1555-1561. https://doi.org/10.3168/jds.S0022-0302(96)76517 -7 .

Yajuvendra, S., S. S. Lathwal, N. Rajput, T. V. Raja, A. K. Gupta, T. K. Mohanty, A. P. Ruhil, A. K. Chakravarty, P. C. Sharma, V. Sharma, and G. Chandra. 2013. Effective and accurate discrimination of individual dairy cattle through acoustic sensing. Appl. Anim. Behav. Sci. 146:11-18. 